\title{
Wireless Sensor Network System for Measuring the Magnetic Noise of Inverter-Fed Three-Phase Induction Motors with Squirrel-Cage Rotor
}

\author{
Andrei Negoita ${ }^{1}$, Gheorghe Scutaru ${ }^{1}$, Ioan Peter ${ }^{2}$, and Razvan Mihai Ionescu ${ }^{1}$ \\ ${ }^{1}$ Transilvania University of Brasov, Advanced Electrical Systems Department, \\ Politehnicii Street No.1, 500036 Brasov, Romania \\ \{Andrei.Negoita, Gheorghe.Scutaru, Razvan.Mihai.Ionescu\} \\ andrei.negoita@yahoo.com \\ ${ }^{2}$ S.C. Electroprecizia S.A., Motor Design Department, \\ Parcului Street No. 18, 505600 Brasov, Romania \\ \{Ioan.Peter\}pr_mot@electroprecizia.ro
}

\begin{abstract}
The object of this paper is the study of the noise produced by inverterfed three-phase induction motors with squirrel-cage rotor. A wireless sensor network based measurement system is proposed, which gives the possibility of measuring the sound pressure virtually simultaneously in multiple points around the motor. In the case of inverter fed motors, the phenomena that lead to the production of the magnetic noise become more complex and the motor becomes noisier because of the increased possibility of matching the exciting frequencies with stator natural frequencies. In order to evaluate the influence of the switching frequency of the PWM inverter on the overall motor noise, the noise-frequency level diagrams (spectrograms) have been traced for a two speed motor of 1.5/2.4 $\mathrm{kW}, 750 / 1500 \mathrm{rpm}$, with 36 stator slots and 46 rotor slots.
\end{abstract}

Keywords: wireless sensor network, induction motor, squirrel-cage, noise.

\section{Introduction}

One of the main sources of noise in a rotating electrical machine is the vibrations excited by electromagnetic forces acting in the motor air-gap. The use of inverters for controlling the speed of induction motors leads to an increase in the harmonic content of the supply voltage. As a consequence, the harmonic content of the air-gap flux increases, thus creating a larger number of magnetic force waves. If the natural frequencies of the motor structure match the frequencies of the magnetic forces, the resonance phenomenon appears which contributes greatly to an increase of the overall noise level of the motor $[1,2]$.

\section{Contribution to Sustainability}

In our days, inverter fed induction motors are frequently used in the residential field. As contribution to the sustainable development, design techniques are required to assure a reduced noise pollution level. 
The object of this paper is the study of the noise produced by inverter-fed threephase induction motors with squirrel-cage rotor. A wireless sensor network system is proposed for measuring the sound pressure virtually simultaneously in multiple points around the motor. The measuring system can be implemented by using low-cost, low power sensors such as miniaturized condenser microphones.

A solution for implementing a system containing a large number of sensors requires the development of a wireless sensor network (WSN) [3]. The flexibility, fault tolerance, high sensing fidelity, low-cost and rapid deployment characteristics of such WSN makes them an ideal platform for condition monitoring of electrical machines[4]. WSN have been applied to condition monitoring of induction motors, either by using the motor current spectral analysis technique which requires the stator current to be sampled and collected [5] or by monitoring bearing vibrations by wireless accelerometers [6].

The IEEE 802.15.4 communication protocol, allows small, power efficient and inexpensive solutions to be implemented for a wide range of devices [7], such as wireless sensors. The protocol can be used for implementing WSN architectures for low cost applications with data throughput as a second consideration. The IEEE 802.15.4 protocol is intended to address applications wherein existing wireless solutions are too expensive or difficult to implement. Table 1 compares the performance of different wireless technologies.

Table 1. A comparison of the 802.15.4 standard with other wireless technologies

\begin{tabular}{llll}
\hline & $802.11 \mathrm{~b}$ & 802.15 .1 & 802.15 .4 \\
& WLAN & Bluetooth & ZigBee \\
\hline Range $[\mathrm{m}]$ & 100 & $10-100$ & 10 \\
Data Throughput $[\mathrm{Mbps}]$ & 11 & 1 & 0.25 \\
Complexity & High & High & Low \\
Cost & High & Medium & Low \\
\hline
\end{tabular}

The 802.15.4 standard allows the formation of two possible network topologies: the star topology and the peer-to-peer topology as seen in Figure 1.

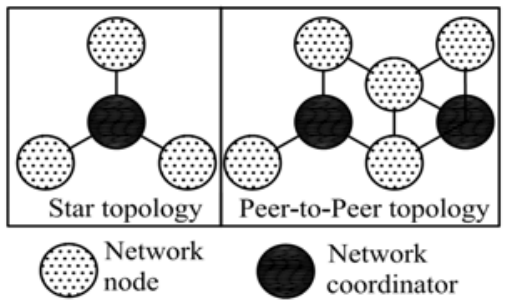

Fig. 1. IEEE 802.15.4 network topologies

One of the main problems concerning the implementation of a WSN network consists in finding the best possible way in which to combine the diverse and sometimes conflicting data gathered by the nodes. Multi-sensor information fusion 
can increase measurement credibility thus improving system reliability. There are many methods employed in multi-sensor data fusion such as [8]: Kalman filter, Bayes estimation, fuzzy set theory and neural networks. The implementation of a Kalman filter approach in the case of the proposed measurement system will be discussed in a future paper.

For the proposed system, a ZigBee protocol WSN was implemented using the Microchip Stack for the ZigBee Protocol. The protocol uses the IEEE 802.15.4 specification as its Medium Access Layer (MAC) and Physical Layer (PHY). According to the IEEE 802.15.4 standard, three types of devices exist in a network.

Their main functions are summarized in Table 2.

Table 2. ZigBee and IEEE 802.15.4 standard device types

\begin{tabular}{lcl}
\hline $\begin{array}{c}\text { ZigBee } \\
\text { device type }\end{array}$ & IEEE device type & \multicolumn{1}{c}{ Network function } \\
\hline Coordinator & $\begin{array}{c}\text { Full Function } \\
\text { Device }\end{array}$ & $\begin{array}{l}\text { Forms the network, allocates network } \\
\text { addresses or allows other devices to join the } \\
\text { network }\end{array}$ \\
Router & $\begin{array}{c}\text { Full Function } \\
\text { Device }\end{array}$ & $\begin{array}{l}\text { Optional device which extends the physical } \\
\text { range of the network or performs } \\
\text { monitoring and control functions. }\end{array}$ \\
End & $\begin{array}{c}\text { Full Function or } \\
\text { Reduced Function } \\
\text { Device }\end{array}$ &
\end{tabular}

As seen in Figure 1, depending on the chosen topology, the elements of a WSN network can communicate with each other directly or through the coordinator. There are two possible types of multi-access mechanisms: beacon and non-beacon. In a nonbeacon enabled network, all nodes in the network are allowed to transmit at any time if the channel is idle. In a beacon enabled network, nodes are allowed to transmit in predefined time slots only. On power-up, the protocol coordinator, based on the number of networks found on each allowed channel, establishes its own network and selects a unique 16-bit Personal Area Network identification. Once a new network is established, protocol routers and end devices are allowed to join the network.

For the proposed system a star topology, $2.4 \mathrm{GHz}, 250 \mathrm{kbps}$, non-beacon, network using a single protocol coordinator was implemented using the Microchip Stack for the ZigBee Protocol.

A sound measurement WSN sensor node consists of a signal conditioning circuit, a microcontroller for data acquisition and conversion, a memory module for data storage and a wireless communication module. Thus the node has both sensing and communication capabilities. The simplified schematic is shown in Figure 2.

The signal conditioning block filters and amplifies the signal coming from the condenser microphone. An inverter amplifier is implemented, using R4 and R5 to set the amplifier offset voltage and R3 and R2 to control the gain. R6 and C4 form an anti-alias low pass filter with a cut-off frequency of $38 \mathrm{kHz}$. 


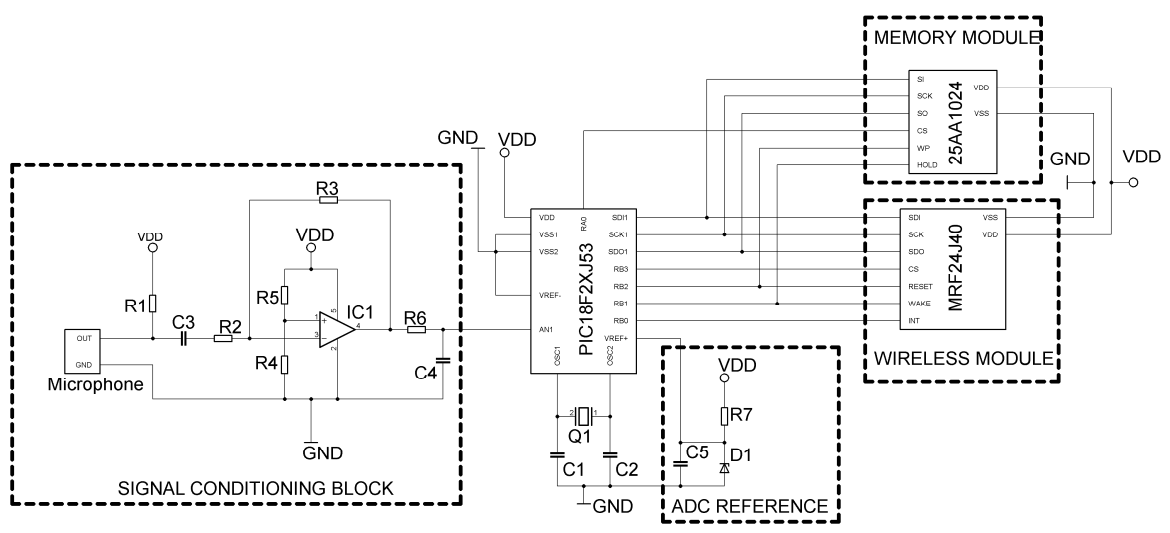

Fig. 2. WSN sensor node simplified schematic

One of the main concerns when signal processing is involved is the aliasing effect, i.e. frequency components of the acquired analog signal greater than half the sample rate of the analog-to-digital converter that shift into the frequency band of the output signal, thus distorting it. Therefore, the sampling frequency of the PIC18F27J53 microcontroller analog-to-digital module must be at least twice the highest frequency of interest of the sampled signal. In our case the sampling frequency was set at $77 \mathrm{kHz}$.

As shown in Figure 3, the microcontroller uses a 12 bit analog-to-digital module for converting the analog signal. The data is then stored into a 1Mbit serial EEPROM memory. The data acquisition process continues as long as the duration of the measurement process set by the user has not been achieved. When the measurement process has ended, the microcontroller begins reading the stored data and sending it to the network coordinator by using the MRF24J40 wireless module.

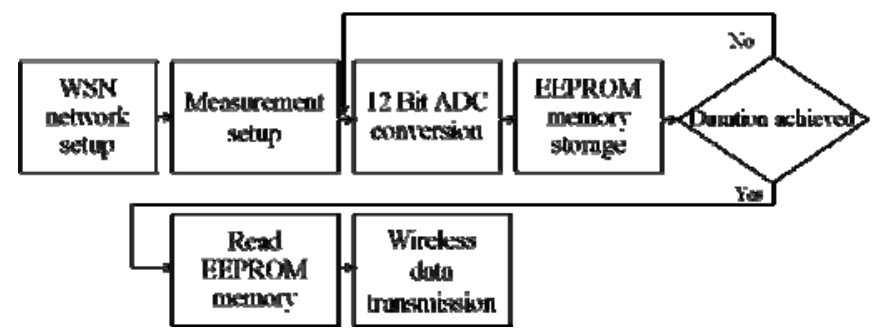

Fig. 3. Data processing and transmission flow-chart

A WSN receiver node, in our case, the network coordinator, receives data sequentially from each of the sensor nodes on the network. As each node transmits, the data is converted by the interface microcontroller and sent to a PC for processing.

The sound measurements were conducted in a semi-anechoic room in compliance with the ISO 1680/1 standard. The WSN nodes can be placed around the motor, on the measurement positions defined by the ISO 1680/1 standard. The measurement setup is shown schematically in Figure 4. 


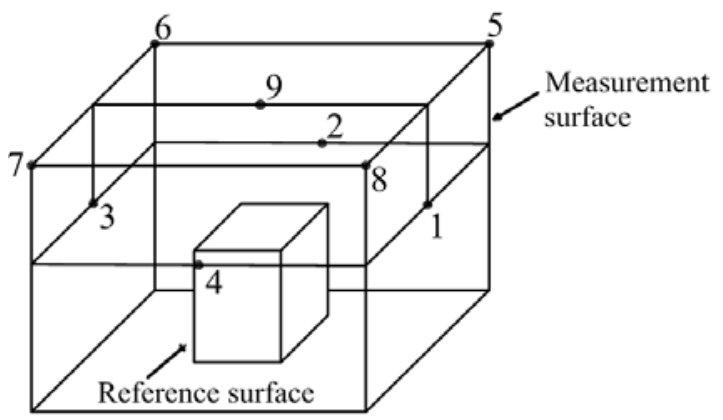

- Measurement point

Fig. 4. Measurement points around the motor according to ISO 1680/1 standard

\section{The Magnetic Noise of Inverter-Fed Induction Motors}

Inverter-fed induction motors are noisier than those fed with sinusoidal current because of the increased possibility of matching the magnetic forces exciting frequencies with stator natural frequencies. The order of the stator current harmonics of an inverter-fed three phase induction motor is:

$$
n=6 k \pm 1
$$

By neglecting the tangential component of the magnetic flux density, according to the Maxwell stress tensor, the magnetic pressure waveform at any point of the air gap can be expressed as:

$$
p_{r}(\alpha, t)=\frac{b^{2}(\alpha, t)}{2 \mu_{0}}=\frac{1}{2 \mu_{0}}\left[b_{1}(\alpha, t)+b_{2}(\alpha, t)\right]^{2}
$$

In terms of Fourier series, the following groups of magnetic waves are produced [1]:

- $\quad p_{r v n}(\alpha, t)$, determined by the product $\left[b_{1}(\alpha, t)\right]^{2}$ of the stator harmonics having the same order $v$. The frequency of the radial magnetic pressure is $f_{r n}=2 n f$ and the vibration mode $r=2 \vee p$.

- $\quad p_{r \mu n}(\alpha, t)$, determined the product $\left[b_{2}(\alpha, t)\right]^{2}$ of the rotor harmonics having the same order $\mu$. The frequency of the radial magnetic pressure is $f_{r n}=2 n f_{\mu}$ and the vibration mode $r=2 \mu p$.

- $\quad p_{r v \mu_{n}}(\alpha, t)$, determined by the interaction of stator harmonics having the order $v$ and rotor harmonics having the order $\mu$.The frequency of the radial magnetic pressure is $f_{r n}=n\left(f \pm f_{\mu}\right)$ and the vibration mode $r=(v \pm \mu) p$.

Higher time stator harmonics of different numbers can produce significant radial forces [2] having the frequency $f_{r n}=\left(n^{\prime} \pm n^{\prime \prime}\right) f ; n^{\prime} \neq n^{\prime \prime}$ and the vibration mode 
$r=0$ or $r=0$. The most important are the magnetic forces due to sums and differences of the fundamental harmonic $f$ with higher order time harmonics of the stator current [1]:

$$
f_{r n}=(1 \pm n) f
$$

The inverter switching frequency has an important effect as the interaction of switching frequency harmonics and higher time harmonics, produces forces with frequencies $f_{n}=n^{\prime} f_{s w} \pm n^{\prime \prime} f$. If n' is an odd integer, $\mathrm{n}$ ', will be an even integer and vice versa:

$$
f_{n}=f_{s w} \pm 2 f, \ldots \quad \text { or } \quad f_{n}=2 f_{s w} \pm f, \ldots
$$

Significant vibration can result from the interaction of permeance field harmonics and MMF harmonics associated with higher time harmonics of the stator [2]:

$$
f_{r n}=\left|f_{n} \pm f\left[1+k \frac{s_{2}}{p}(1-s)\right]\right|
$$

and the vibration mode $r=0,2$.

In the case of inverter-fed motors, the magnetic component of noise is modified. In order to evaluate this modification, a two speed, $1.5 / 2.4 \mathrm{~kW}, 750 / 1500 \mathrm{rpm}$ motor, with 36 stator slots and 46 rotor slots was tested. The spectrograms have been traced using a Bruel \& Kjaer 2112 spectrum analyzer.

The motor was tested for no load operation and was supplied from the network or a "Telemecanique" ATV-58HU54N4 inverter with a switching frequency set at $2 \mathrm{kHz}$. The results obtained for the $1500 \mathrm{rpm}$ speed are shown in Figure 5 and 6.

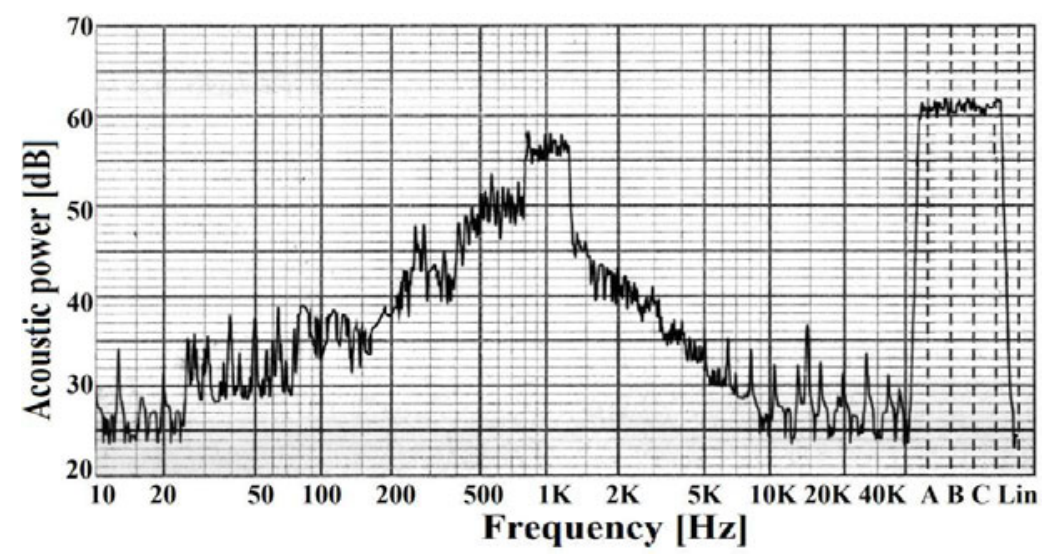

Fig. 5. No load operation, $U_{N}=400 \mathrm{~V}$ and $50 \mathrm{~Hz}$, network supplied, $2.4 \mathrm{~kW}-1500 \mathrm{rpm}$ 


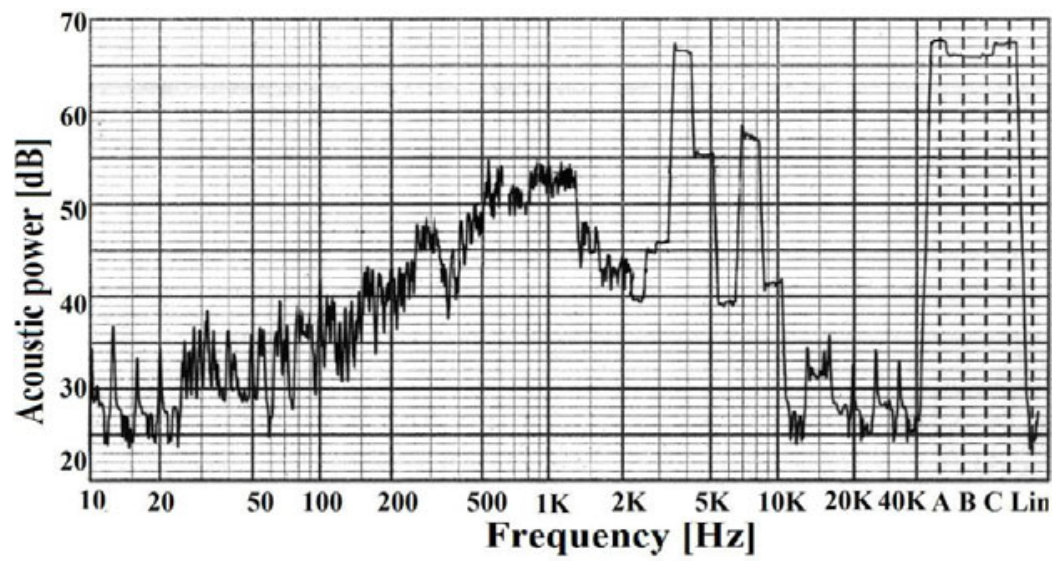

Fig. 6. No load operation, $U_{N}=400 \mathrm{~V}$ and $50 \mathrm{~Hz}$, inverter supplied, $2.4 \mathrm{~kW}-1500 \mathrm{rpm}$

For the $1500 \mathrm{rpm}$ speed, two new noise peaks appear between 2500 and $4000 \mathrm{~Hz}$. and 7000 and $8000 \mathrm{~Hz}$. The noise increases by $7 \mathrm{~dB}$.

\section{Conclusions and Future Work}

The presented spectrograms will be used as a reference for evaluating the performance of the proposed wireless sound measuring system.

The applicability of the system depends on several factors. Battery life is extremely important, as most power is used for wireless communication. In order to maximize battery life, the system must take advantage of the sleep mode operation feature of the microcontroller and the wireless communication modules.

The overall speed and accuracy of the proposed system can be significantly improved by using larger data throughput protocols like Bluetooth and superior microprocessors like Digital Signal Processors. From the implementation point of view, this could eliminate the need for an external EEPROM memory and would provide real time sound measurements capability. However, these improvements would lead to significant increase of system cost and development time.

\section{Acknowledgement}

This paper is supported by the Sectoral Operational Programme Human Resources Development (SOP HRD), ID59321 financed from the European Social Fund and by the Romanian Government.

\section{References}

1. Scutaru, G., Peter, I.: The noise of electrical induction motors. LUX Libris Publishing House, Brasov (2004) (in Romanian)

2. Gieras, J., Wang, C., Cho Lai, J.: Noise of polyphase induction motors. Taylor \& Francis, Abington (2006) 
3. Yick, J., Mukherjee, B., Ghosal, D.: Wireless sensor network survey. Computer Networks 52, 2292-2330 (2008)

4. Korkua, S., Jain, H., Lee, W., Kwan, C.: Wireless Health Monitoring System for Vibration Detection of Induction Motors. In: IEEE Industrial and Commercial Power Systems Technical Conference, pp. 1-6 (2010)

5. Lu, B., Wu, L., Habetler, T.G., Harley, R.G., Gutierrez, A.T.: On the Application of Wireless Sensor Networks in Condition Monitoring and Energy Usage Evaluation for Electric Machines. In: 31st Annual Conference of the IEEE Industrial Electronics Society, pp. 2674-2679 (2005)

6. Jagannath, V.M.D., Raman, B.: WiBeaM: Wireless Bearing Monitoring System. In: 2nd Int. Conf. on Communication Systems Software and Middleware, pp. 1-8 (2007)

7. IEEE 802.15.4 Standard for Information Technology - Telecommunications and information exchange between systems - Local and metropolitan area networks - Specific requirements, http://standards.ieee.org/getieee802/802.15.html

8. Zhou, H.: Multi-sensor Information Fusion Method Based on the Neural Network Algorithm. In: Fifth International Conference on Natural Computation, ICNC 2009, pp. 534-536 (2009) 